\title{
Circulating miR-21 and TGF- $\beta 1$ expression levels in patients with cardiovascular diseases
}

\author{
Irina Huica ${ }^{1}$, Iulia V. Iancu ${ }^{1}$, Anca Botezatu ${ }^{1}$, Otilia Banu², Elena Lupeanu, Mariana Anton ${ }^{4}$
}

Corresponding author:

Irina Huica, MD

"Stefan S Nicolau" Institute of Virology

Bucharest, Romania

\author{
"Stefan S Nicolau" Institute of Virology, Bucharest \\ 2Emergency Institute for Cardiovascular Diseases "Prof Dr. C.C. lliescu", Bucharest \\ ${ }^{3}$ National Institute of Gerontology and Geriatrics "Ana Aslan", Bucharest \\ 4"Coltea" Clinical Hospital, "Carol Davila" University of Medicine and Pharmacy, Bucharest
}

\section{ABSTRACT}

Recent works focus on the role microRNAs (miRNAs) play in regulation of cardiovascular pathology. One of the miRNAs highly expressed in cardiovascular system is miR-21.

The aim of this study was to investigate the relationship between the expression level of miR-21 in cardiac disease and correlate with TGF- $\beta 1$, one of its targets.

Methods: Plasma samples from patients with CVD (cardiovascular diseases) established according to clinical and paraclinical exams were subjected to total RNAs isolation. miR-21 expression levels in patients with CVD and in healthy control subjects were quantified on $A B I$ 7300 real-time PCR (Applied BioSystems) and normalized to RNU-43. TGF- $\beta 1$ was predicted as potential target of miR-21 using MicroCosm TARGETS and quantified in TaqMan system (normalized to GAPDH). Statistical analysis was performed with GraphPad Prism.

Results: Significant lower levels of miR-21 were found in CVD patients vs. controls $(p<0.0001)$. An inverse correlation between miR-21 and TGF- $\beta 1$ expression levels was noted in CVD patients. miR-21 levels vs. control were significantly different for ATHE, CAD and ATH groups ( $p=0.0016,0.0058$ and 0.0032 respectively) and correlated with TGF- $\beta 1$ expression and cardiac overload.

Conclusions: The results of this study suggest a good correlation between miR-21 and TGF- $\beta 1$ in cardiovascular diseases.

Key words: miR-21, TGF- $\beta 1$, cardiovascular disease

\section{INTRODUCTION}

Cardiovascular diseases represent the major cause of morbidity and mortality in developed countries. Their development involve genetic and epigenetic alterations that were object of many studies in the past decades.

miRNAs have emerged as novel gene regulators including pathways associated with cardiovascular pathology (1). MicroRNAs (miRNAs) are a class of naturally occurring small noncoding RNAs. They bind to the complementary target sites in 3'-untranslated regions and supress targeted mRNA through translational inhibition or transcriptional degradation $(2,3)$. Each mRNA can be 
targeted by several miRNAs and each miRNA might target different mRNAs. As more than $30 \%$ of genes are regulated by this epigenetic mechanism, miRNAs are implied in normal development and pathogenesis (4). miRNAs might modulate various pathophysiological mechanisms of CVD, the most important being atherosclerosis. In atherosclerosis, miRNAs exert a dual role, this making them potential diagnostic and prognostic biomarkers, especially in coronary pathology. Targeting miRNAs might become a new strategy for the treatment of CVD.

Recent studies show that miR-21 is highly expressed in cardiovascular system, its levels being deregulated under CVD conditions (5). In animal model, it was reported down-regulated expression levels of miR-21 in infarcted hearts while an upregulated expression in border areas of the infarct (5) correlated to a protective effect against ischemia-induced cardiac cell death. Also, Yin et al. demonstrated that myocardial infarct size was significantly reduced after treatment with heatshock- or preconditioning-induced miRNAs including miR-21 $(6,7)$. Still, there are controversial data about miR-21 levels in cardiac diseases, probably because different types of samples (tissue, blood) were used or depending on the time point at which samples are taken (8).

Hemodinamic overload caused by chronic hypertension, atheromatosis or chronic myocardial infarction leads to adaptative remodeling of the heart, a complex process that can include cardiomyocyte hypertropy, fibrosis, EMC deposition. At molecular level, TGF- $\beta 1$ is one of the key mediators of these processes. In cardiac tissue repair, new connective tissue needs to be formed, this being achieved by fibroblasts migration and proliferation in the lesion. TGF- $\beta 1$ induces fibroblasts to produce and remodel ECM (extracellular matrix) resulting in healing the wound (9). This cytokine is released especially by platelets (10) and is expressed in high concentrations in inflammatory and prothrombotic states. Increased expression of TGF- $\beta 1$ was found in human atherosclerotic plaques (11) and in heart failure (12), while in the overloaded human heart its up-regulation is directly dependent by the fibrosis degree (13). Studies conducted on transgenic mice had shown that TGF- $\beta 1$ overexpression leads to interstitial fibrosis and hypertrophic growth of cardiac myocytes (14).

Multiple miRNA target prediction tools are now available. Using MicroCosm TARGETS software, ver. 5, (TargetScan, ver. 7) TGF- $\beta 1$ (transforming growth factor- $\beta 1$ ) was proposed as a predicted target of miR-21.
The aim of this study was to investigate miR-21 circulating levels in patients with cardiovascular diseases and correlate with TGF- $\beta 1$ expression.

\section{MATERIALS AND METHODS}

\section{Study group}

59 subjects, aged $23-83$ years old (mean $=53.6)$ were selected from patients with CVD treated in "Coltea" Clinical Hospital and Institute of Geriatric and Gerontology "Ana Aslan" during 2011-2013. The patients were selected based on clinical and paraclinical exams (EKG, coronarography, TTE - transthoracic echocardiogram, Holter EKG, MATA, ABI and metabolic profile). Also, risk factors like smoking, blood pressure, lipid and glycemic profiles were analyzed. A control group consisting in subjects without CVD ( $n=21$, aged 18 - 69 years old, mean $=47.9$ ) was included.

From each enrolled subject blood samples were collected in EDTA sprayed tubes after written informed consent was obtained. Plasma samples were obtained following centrifugation at $700 \mathrm{rpm}$ for 6 minutes.

\section{Total RNA isolation}

Total RNA was isolated from plasma samples using Trizol reagent (Invitrogen) according to manufacturer instructions. After on-column DNase I treatment with RNase-free DNase (Qiagen), RNA quantity and quality were determined using a NanoDrop Technologies ND1000 spectrophotometer and Experion Analyser (BioRad) (RIN >7.5). Samples were stored at $-80 \circ \mathrm{C}$ and used to generate cDNAs.

\section{miR-21 quantification}

The expression levels of miR-21 in patients with CVD and healthy control subjects were quantified using TaqMan MicroRNA assays (Applied Biosystems). $10 \mathrm{ng}$ total RNA were used for miRNA specific reverse transcription according to manufacturer protocol: each sample was incubated 30 minutes at $16 \% \mathrm{C}, 30$ minutes at $42^{\circ} \mathrm{C}$, than 5 minutes at $85^{\circ} \mathrm{C}$. The reverse transcription reaction was performed at $37{ }^{\circ} \mathrm{C}$ for 1 hour followed by $5 \mathrm{~min}$ at 95 으 for enzyme denaturation.

miR-21 expression levels were quantified on $A B I$ 7300 real-time PCR (Applied BioSystems) and normalized to RNU43. RNU43 has demonstrated both stable and abundant expression across different human tissues and organs; it is regarded as one of the controls with the least variability for miRNAs assays and has been widely used in different fields (15). Each reaction was performed in a final volume of $25 \mu \mathrm{l}$ containing $5 \mu \mathrm{l}$ cDNA (10ng), $12.5 \mu \mathrm{l}$ TaqMan Universal PCR Master Mix 
(No AmpErase) and $1.25 \mu \mathrm{l}$ TaqMan miRNA probe (Applied BioSystems).

The thermal cycle was set as follows: $10 \mathrm{~min}$ template denaturation at $95{ }^{\circ} \mathrm{C}, 40$ cycles of denaturation at $95^{\circ} \mathrm{C}$ for $15 \mathrm{sec}$ and primer annealing/elongation at $60^{\circ} \mathrm{C}$ for $1 \mathrm{~min}$.

\section{Bioinformatic tools}

MicroCosm TARGETS software, version 5 (16) was used to find miR-21 mRNA predictive targets. This program uses miRanda algorithm to find potential binding sites for a given miRNA and verifies the complementarity at the $5^{\prime}$ end of the miRNA throwing away alignments with more than one base not complementary with a target site. Targets are passed through Vienna RNA folding routines to estimate the thermodynamic stability. TGF- $\beta 1$ was found to be a potential target of miR-21 having a Pct $=0.83$ (probability of conserved targeting - according to Friedman et al 2009 (17).

\section{TGF- $\beta 1$ quantification}

For estimating TGF- $\beta 1$ (predicted target gene) expression, total RNA was reverse transcribed using oligo-dT primers (Promega). Briefly, $2.5 \mu \mathrm{g}$ RNA, $1 \mu \mathrm{l}$ oligo-dT $(0,5 \mu \mathrm{g} / \mu \mathrm{l})$ and $1 \mu \mathrm{l}$ dNTPs $(10 \mathrm{mM})$ were incubated 5 minutes at $65^{\circ} \mathrm{C}$. After cooling on ice, $4 \mu \mathrm{l}$ RT buffer (5x), $2 \mu \mathrm{l}$ DTT (200 mM) and 1 $1 \mu$ l RNAse inhibitor (40U) were added. After 2 minutes at 37으, 1 $\mu \mathrm{l}$ of M-MLV (200U) reverse transcriptase was added and the samples were incubated for 60 minutes at $37{ }^{\circ} \mathrm{C}$ and afterwards 15 minutes at $70 \circ \mathrm{C}$. cDNAs were stored at $-20^{\circ} \mathrm{C}$ until use.

TGF- $\beta 1$ levels were quantified on ABI 7300 real-time PCR (Applied BioSystems) and normalised to GAPDH. Each reaction was performed in a final volume of $25 \mu \mathrm{l}$ containing $5 \mu \mathrm{l}$ cDNA (100 ng), $12.5 \mu \mathrm{l}$ TaqMan Universal PCR Master Mix (No AmpErase) and 1.25 $\mathrm{l}$
TaqMan TGF- $\beta 1$ probe (Applied BioSystems).

To check for amplicon contamination, every run contained a negative control in which nuclease-free water was a substitute for template. Samples were tested in duplicate.

\section{Statistical analysis}

Data collected were analysed using Graph Pad Prism software (Graph Pad Software Inc., San Diego, CA, USA) for the statistical analyses. For each group, the values were expressed as mean, median, minimum and maximum, and standard error. One-way ANOVA (and non-parametric) test was used for analysing the groups and distinguish the significant differences and MannWhitney test (non-parametric) values for comparing each case group with controls. $P$ values $<0.05$ were considered significant. Linear regression and Spearman correlation were used to investigate the correlation degree between the expression levels of mir-21 and TGF- $\beta 1$.

\section{RESULTS}

The physical and physiological characteristics of the patients and controls are presented in table 1. No significant differences $(p>0.05)$ between cases and controls were observed for sex, age, smoking, HDL and diastolic blood pressure.

Higher values were obtained for fasting glucose, total cholesterol and LDL ( $p=0.0112,0.0057$ and 0.0186 respectively), while values for HDL were similar $(p=0.1301)$.

Analyzing the circulating levels of miR-21 we found significant differences between patients and controls $(p=0.0003)$. Assessing TGF- $\beta 1$ levels, differences between groups were significant $(p=0.0079)$.

Linear regression test between TGF- $\beta 1$ and miR-21 $\mathrm{n}$-fold values was performed (figure 2 ) in order to

Table 1. Patients' characterization. * $=$ significant $\mathrm{p}$

\begin{tabular}{|c|c|c|c|}
\hline & CASE & CONTROL & $P$ (case / control) \\
\hline number of cases & 59 & 21 & - \\
\hline men / women & $27 / 32$ & $12 / 9$ & 0.3767 \\
\hline age, [years] & $53.6(23-83)$ & $47.9(18-69)$ & 0.1014 \\
\hline smokers $[\mathrm{y} / \mathrm{n}]$ & $21 / 38$ & $11 / 10$ & 0.1823 \\
\hline Fasting glucose, $[\mathrm{mg} / \mathrm{dl}]$ & $108.1(78-259)$ & $89.6(81-102)$ & $0.0112^{\star}$ \\
\hline $\mathrm{CHOL}[\mathrm{mg} / \mathrm{dl}]$ & $195.9(114-293)$ & $150.6(80-188)$ & $0.0057^{*}$ \\
\hline $\mathrm{HDL}[\mathrm{mg} / \mathrm{dl}]$ & $42.9(33.0-68.0)$ & $47.3(26.8-57.1)$ & 0.1301 \\
\hline $\mathrm{LDL}[\mathrm{mg} / \mathrm{dl}]$ & $125.1(83.5-168.7)$ & $92.7(87.0-99.1)$ & $0.0186^{\star}$ \\
\hline Systolic blood pressure (mm Hg) & $138.1(100.0-190.0)$ & $116.6(95.0-135.0)$ & $P<0.0001^{\star}$ \\
\hline Diastolic blood pressure (mm Hg) & $75.16(55.0-100.0)$ & $70.1(55.0-90.0$ & 0.1307 \\
\hline
\end{tabular}



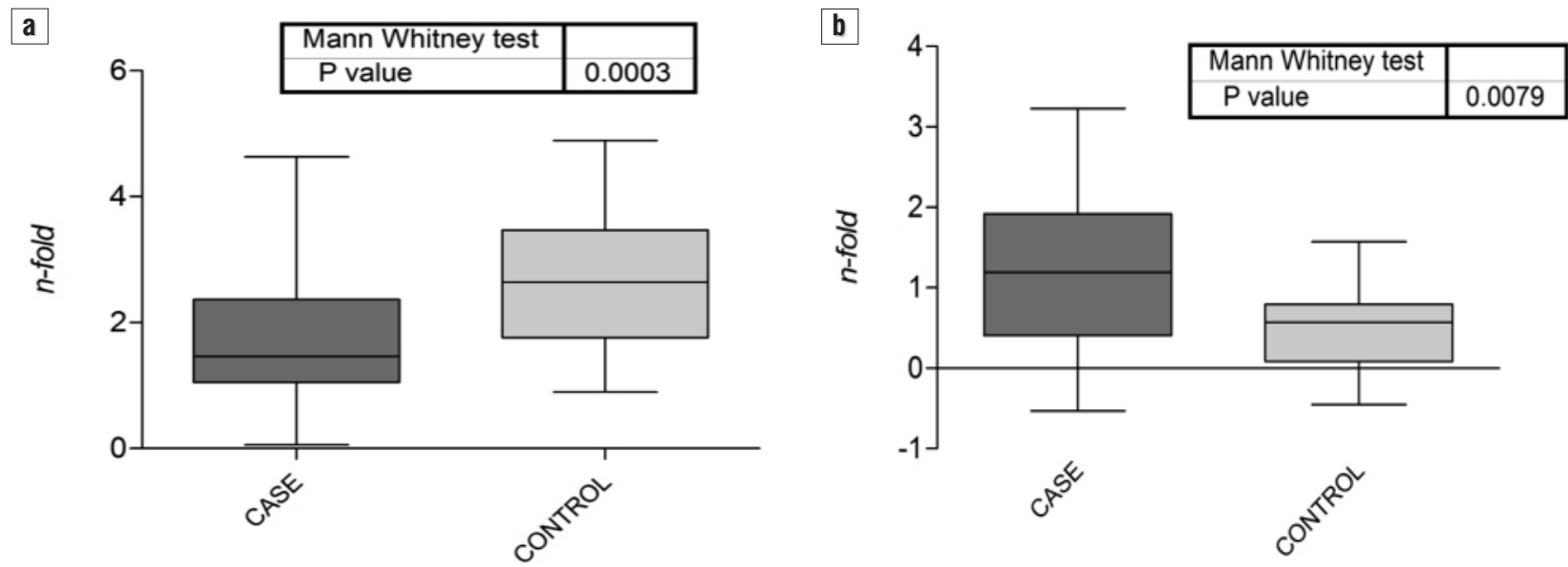

Figure 1. The expression levels of miR-21 (a) and TGF- $\beta 1$ (b) in patients and controls

verify the cytokine as a potential target of this miRNA.

An inverse correlation between the investigated parameters was observed as the test returned a good correlation coefficient $\left(r^{2}=0.3753\right)$.

Based on cardiovascular diagnosis and according with ESC guides, the patients were divided into 4 distinct groups: (1) atheromatosis (ATHE) (2) coronary artery disease - CAD (3) cardiomyopathy (CM) and (4) arterial hypertension (AHT). The patients in ATHE group presented Ankle-Brachial Index $(A B I)<0.7$, those with CAD displayed $>50 \%$ coronary stenosis and the subjects with CM had $<40 \%$ LVEF.

ANOVA test was performed to compare the expression levels of miR-21 and TGF- $\beta 1$ in the four groups of patients. miR-21 and TGF- $\beta 1$ significantly differences among the studied groups were noted $(p<0.0001$ and $p=0.0002$ respectively) (figure 3 ).

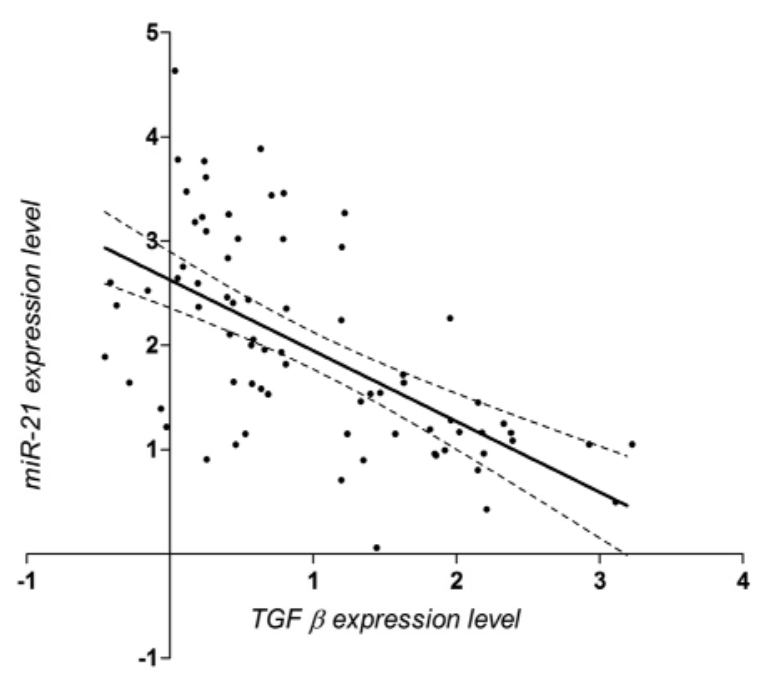

Figure 2. Correlation between miR21 and TGF- $\beta 1$ a

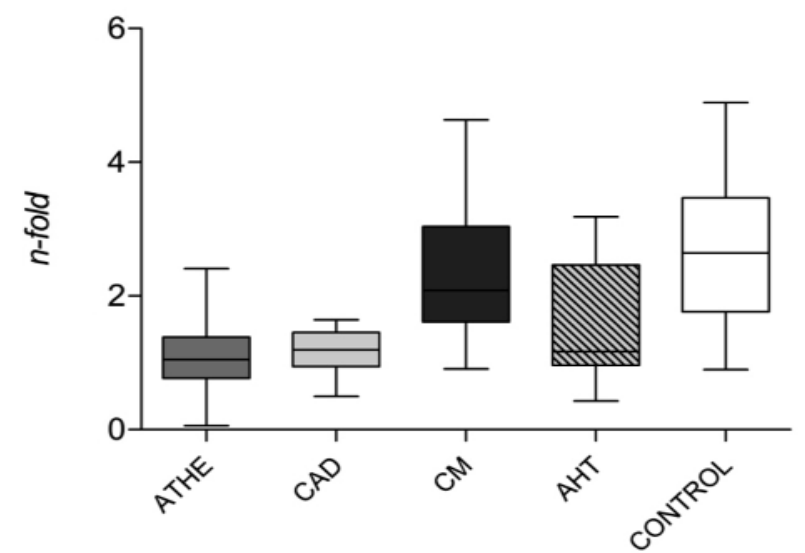

b

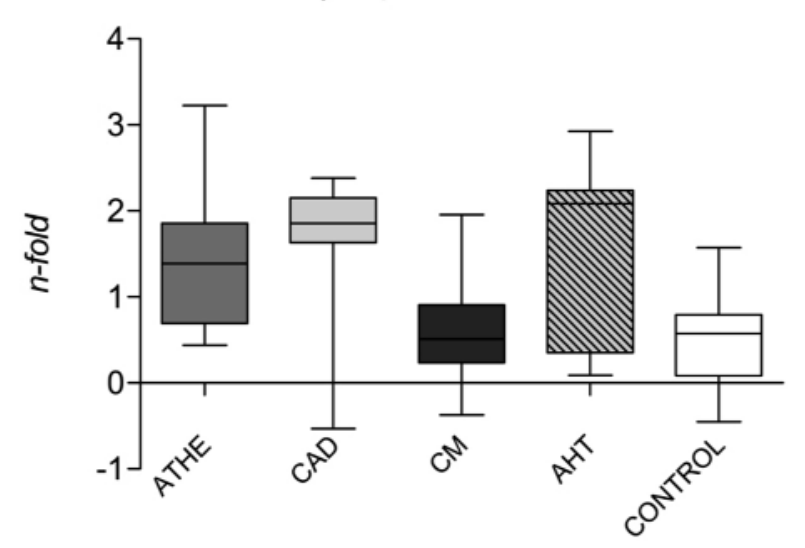

Figure 3. miR-21 (a) and TGF- $\beta 1$ (b) expression levels in cases and controls 
miR-21 expression levels vs. control were significantly different for ATHE, CAD and ATH groups $(p=$ $0.0016,0.0058$ and 0.0032 respectively).

For TGF- $\beta 1$, increased values were found in ATHE, $C A D$ and $A H T$ groups, this sustaining that TGF- $\beta 1$ over-expression is correlated with cardiac overload. Significant differences were noticed for ATHE $(p<$ $0.0001), C A D(p=0.0017)$ and $A H T(p=0.0021)$ groups when compared with controls (table 2).

Aging is accompanied by remodeling of the regulatory pathways, including miRNAs. The levels of the circulating miRNAs are suggested to display an agedependent pattern that can modulate either the longevity of processes they control, or age-related diseases (18). On the other hand, TGF- $\beta$ levels also correlates with aging and age-related diseases (CVD). Therefore, in our study we investigated the correlation between subjects' age vs. miR-21 and TGF- $\beta 1$ respectively (figure 4).

We found lower miR-21 levels in aged patients, and, as a consequence, TGF- $\beta$ presented higher values, regardless the cardiovascular conditions. Spearman correlation returned -0.5058 for miR-21 and 0.5501 for TGF- $\beta 1$.

\section{DISCUSSION AND CONCLUSIONS}

Most miRNAs linked to CVD display altered expressions as a consequence of the complex miRNAs network that regulate the cardiac physiologic processes. Among these miRNAs, miR-21 has been shown to play important role in development of heart disease. Several studies reported a protective effect of miR-21 shortly post AMI (acute myocardial infarct) (19, 20) and in dilated, ischemic and hypertrophyc cardiomiopathy with heart failure (21). In our study, CM patients presented higher levels of miR-21 compared with CAD, ATHE and AHT groups and lower levels comparing with controls.

The rupture of atheroma fibrous cap leads to abnormal miR-21 and miR-24 levels that together regulate the matrix metalloproteinases components of the fibrous head. On the other hand, in vitro TGF- $\beta 1$ activates endothelial matrix metalloproteinase expres-

Table 2. miR-21 and TGF- $\beta 1$ expression levels (n-fold reported to house-keeping RNU-43 and GAPDH respectively)

\begin{tabular}{|c|c|c|c|c|c|c|}
\hline & & ATHE & CAD & CM & AHT & CONTROL \\
\hline \multirow[t]{6}{*}{ miR-21 expression level (n-fold) } & Mean & 1,096 & 1,167 & 2,274 & 1,540 & 2,561 \\
\hline & Median & 1,050 & 1,195 & 2,082 & 1,167 & 2,564 \\
\hline & Minimum & 0,05867 & 0,4963 & 0,9053 & 0,4262 & 0,8979 \\
\hline & Maximum & 2,408 & 1,641 & 4,633 & 3,181 & 4,778 \\
\hline & Std. Error & 0,1714 & 0,1397 & $0,1837^{-}$ & 0,2320 & 0,2923 \\
\hline & $P^{-1}$ & 0.0016 & 0.0058 & 0.7081 & 0.0032 & n/a \\
\hline \multirow[t]{6}{*}{ TGF- $\beta$ expression level (n-fold) } & Mean & 1,526 & $1,610^{-}$ & 0,5974 & 1,613 & 0,4859 \\
\hline & Median & 1,388 & 1,858 & 0,5116 & 2,085 & 0,5735 \\
\hline & Minimum & 0,4413 & $-0,5307$ & $-0,3727$ & 0,09121 & $-0,4537$ \\
\hline & Maximum & 3,227 & 2,381 & 1,956 & 2,926 & 1,573 \\
\hline & Std. Error & 0,2638 & 0,3683 & $0,1175^{-}$ & 0,2592 & 0,1156 \\
\hline & $\mathrm{P}$ & $<0.0001$ & 0.0017 & 0.1208 & 0.0021 & $n / a$ \\
\hline
\end{tabular}

${ }^{\star}$ To compare each group with the controls, MANN-WHITNEY test was performed
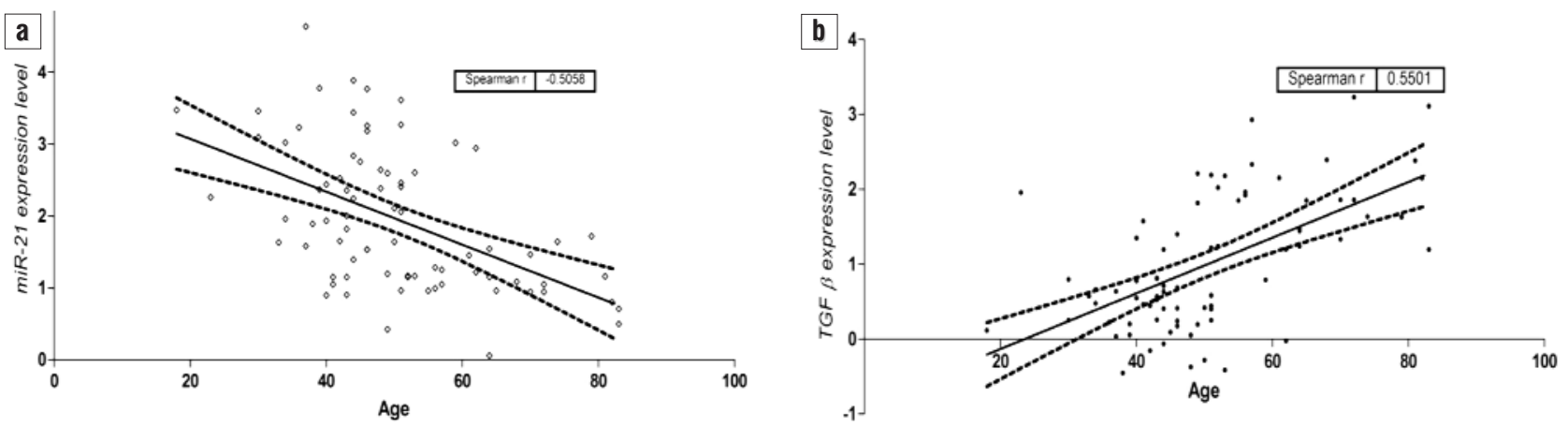

Figure 4. Correlation between miR21 (a) and TGF- $\beta 1$ (b) expression levels and age 
sion and activity via miR-21, this confirming interconnectivity between them (22). Diehl et al noted that CAD patients without AMI display low levels of miR-21 (23). The results are similar to Lacatta et al who noted miR21 downregulation in CAD cases with unstable angina and AMI (24).

We found miR-21 decreased expression levels of miR-21 in ATHE, CAD and AHT groups, as compared with controls. For CAD, the results confirm data of Diehl et al. On the other hand, our data are not concordant with study of Li et al who found increased miR-21 levels in patients with peripheral arterial disease (aterosclerosis obliterans), but their study was performed on tissue samples (25).

In subclinical atherosclerosis miR-21 was found increased (26). In our study, patients enrolled in ATHE group display lower levels of miR-21 vs control.

miR-21 values were correlated with the expression levels of TGF- $\beta 1$. Down-regulation of miR-21 was found in aged patients. F. Olivieri et al reported higher miR-21 values in aging, but on healthy subjects (18). Several studies performed on both human and animal model showed that TGF- $\beta 1$ expression levels increase in stressed cardiac myocytes (27). Our data demonstrate that TGF- $\beta 1$ has increased expression in blood of patients having different heart and circulation conditions, that may stress the myocardium (atheromatosis, coronary artery disease, arterial hypertension), but the values were found to be similar with the controls in the case of patients with cardiomyopathy. In several studies TGF- $\beta 1$ was found particulary overexpressed in ventricular myocardium during transition from stable hypertrophy to heart failure $(28,29)$. The authors considered that TGF- $\beta 1$ mediates the adaptation of heart muscle to overload.

A good correlation was found between expression levels of miR-21 and those of TGF- $\beta 1$ in concordance with the bioinformatics prediction that TGF- $\beta 1$ could be a target of miR-21.

During aging the heart suffers changes like hypertrophy, decreased in left ventricular diastolic and systolic function, arterial rigidity and impaired of endothelial function. Also, apoptotic and necrotic processes decreased cardiomyocytes and increased oxidative stress, leading to proinflammatory status.

The results of this study suggest a good correlation between miR-21 and TGF- $\beta 1$ in cardiovascular diseases.

\section{REFERENCES}

1. Friedman JM, Jones PA MicroRNAs: critical mediators of differentiation, development and disease. Swiss Medical Weekly. 2008;139(33-34): 466-72

2. Pillai RS. MicroRNA function: multiple mechanisms for a tiny RNA? RNA.
2005 Dec;11(12):1753-61.

3. Zamore PD, Haley B. Ribo-gnome: the big world of small RNAs. Science. 2005;309:1519-24

4. Chen LJ, Lim SH, Yeh YT, Lien SC, Chiu JJ .Roles of miRNAs in atherosclerosis and restenosis, J Biomed Sci. 2012 Aug 29;19(1):79-92

5. Cheng Y, Zhang C. MicroRNA-21 in cardiovascular disease. J Cardiovasc Transl Res. 2010;3(3):251-5

6. Yin C, Salloum FN, Kukreja RC. A novel role of microRNA in late preconditioning: upregulation of endothelial nitric oxide synthase and heat shock protein 70. Circ Res. 2009;104(5):572-5.

7. Yin C, Wang X, Kukreja RC. Endogenous microRNAs induced by heatshock reduce myocardial infarction following ischemia-reperfusion in mice. FEBS Letters. 2008;582(30):4137-42

8. Han H, Qu G, Han C, Wang Y, Sun T, Li F et al. MiR-34a, miR-21 and miR23a as potential biomarkers for coronary artery disease: a pilot microarray study and confirmation in a 32 patient cohort. Exp Mol Med. 2015;47.e138; doi:10.1038/emm.2014.81

9. Manabe I, Shindo T, Nagai R. Gene expression in fibroblasts and fibrosis: involvement in cardiac hypertrophy. Circ Res. 2002;91:1103-13.

10. Gasparyan AY, Stavropoulos-Kalinoglou A, Mikhailidis DP, Douglas KM, Kitas GD. Platelet function in rheumatoid arthritis: arthritic and cardiovascular implications. Rheumatol Int. 2011;31(2):153-64.

11. Nikol S, Isner JM, Pickering JG, Kearney M, Leclerc G, Weir L. Expression of transforming growth factor-beta 1 is increased in human vascular restenosis lesions. J Clin Invest. 1992;90(4):1582-92

12. Lim H, Zhu YZ. Role of transforming growth factor-beta in the progression of heart failure. Cell Mol Life Sci. 2006;63:2584-96.

13. Hein S, Arnon E, Kostin S, Schonburg M, Elsasser A, Polyakova et al. Progression from compensated hypertrophy to failure in the pressureoverloaded human heart. Structural deterioration and compensatory mechanisms. Circ. 2003;107:984-91.

14. Brooks WW, Conrad CH. Myocardial fibrosis in transforming growth factor beta(1)heterozygous mice. J Mol Cell Cardiol. 2000;32:187-95.

15. Wong L, Lee K, Russell I, Chen C (2007) Endogenous Controls for Real-Time Quantitation of miRNA Using TaqMan ${ }^{\circledR}$ MicroRNA Assays. Applied Biosystems Application Note, Publication 127AP11-01, available at www.appliedbiosystems.com

16. http://www.ebi.ac.uk/enright-srv/microcosm/htdocs/targets/v5/

17. Friedman RC, Farh KKH, Burge CB, Bartel DP. Most mammalian mRNAs are conserved targets of microRNAs. Genome Res. 2009;19:92-105.

18. Olivieri F, Spazzafumo L, Santini G, Lazzarini R, Albertini MC, Rippo MR et al. Age-related differences in the expression of circulating microRNAs: miR21. Mech Ageing Dev. 2012;133(11-12):675-85

19. Dong $\mathrm{S}$, Cheng $\mathrm{Y}$, Yang J. microRNA expression signature and the role of miR-21 in the early phase of AMI. J Biol Chem, 2009;284:29514-25

20. Gu GL, XU XL, Sun XT, Zhang J, Guo CF, Wang CS et al. Cardioprotective Effect of MicroRNA-21 in Murine Myocardial Infarction. Cardiovasc Therapeutics. 2015;33: 109-17.

21. Latronico MV, Catalicci D, Condorelli G. Emerging role of microRNAs in cardiovascular biology. Circulation Res. 2007;101(12):1225-36.

22. Kumarswamy R, Volkmann I, Jazbutyte V, Dangwal S, Park DH, Thum T. Transforming Growth Factor-Induced Endothelial-to-Mesenchymal Transition Is Partly Mediated by MicroRNA-21. Arterioscler Thromb Vasc Biol. 2012;32:361-9

23. Diehl P, Fricke A, Sander L. Microparticles: major transport vehicles for distinct microRNAs in circulation. Cardiovascular Res. 2012;93:633-44.

24. Lacatta EG, Levy D. Arterial and cardiac aging: major shareholders in cardiovascular disease enterprises: Part 2 the agin hart in health links to heart disease. Circ. 2003:107:346-54

25. Li T, Cao H, Zhuang J. Identification of miR-130a, miR-27b and miR-210 as serum biomarkers for atherosclerosis obliterans. Clin Chim Acta. 2011; 412:66-70.

26. Cenghiz M, Yavuzer S, Kilickiran A. Circulating miR-21 and eNOS in subclinical atherosclerosis in patients with hypertension.Clin Exp Hypertens. 2015;19:1-7

27. Mondragon RR, Galindo CA, Avila G. Role of TGF- $\beta$ on cardiac structural and electrical remodeling. Vasc Health Risk Manag. 2008;4(6):1289-1300

28. Rosenkranz S. TGF- $\beta 1$ and angiotensin networking in cardiac remodeling. Cardiovasc Res. 2004;63:423-32

29. Verheule S, Sato T, Everett T.Increased vulnerability to atrial fibrillation in transgenic mice with selective atrial fibrosis caused by overexpression of TGF-beta 1. Circ Res. 2004:94:1458-65 